\title{
Comparison of adrenaline injection and bipolar electrocoagulation for the arrest of peptic ulcer bleeding
}

\author{
H-J Lin, G-Y Tseng, C-L Perng, F-Y Lee, F-Y Chang, S-D Lee
}

\begin{abstract}
Background-Peptic ulcers with active bleeding or a non-bleeding visible vessel require aggressive endoscopic treatment. Aims-To determine whether endoscopic adrenaline injection alone or contact probe therapy following injection is a suitable treatment for peptic ulcer bleeding.

Methods-A total of 96 patients with active bleeding or non-bleeding visible vessels received adrenaline alone, bipolar electrocoagulation alone, or combined treatment ( $n=32$ in each group).

Results-Initial haemostasis was not achieved in one patient in the adrenaline group, two in the gold probe group, and two in the injection gold probe group $(p>0.1)$. Rebleeding episodes were fewer in the injection gold probe group $(2 / 30,6.7 \%)$ than in the gold probe group $(9 / 30,30 \%$, $\mathbf{p}=0.04$ ) and in the adrenaline group $(11 / 31,35.5 \%, p=0.01)$. Treatment failure (other therapy required) was rarer in the injection gold probe group (4/32, 12.5\%) than in the adrenaline group (12/32, $37.5 \%, p=0.04)$. The volume of blood transfused after entry of the study was less in the injection gold probe group (mean $491 \mathrm{ml}$ ) than in the adrenaline group (1548 $\mathrm{ml}, \mathrm{p}<0.0001)$ and the gold probe group (1105 ml, p<0.01). Duration of hospital stay, numbers of patients requiring urgent surgery, and death rate were not statistically different among the three groups.

Conclusions-For patients with peptic ulcer bleeding, combined adrenaline injection and gold probe treatment offers an advantage in preventing rebleeding and decreasing the need for blood transfusion. (Gut 1999;44:715-719)
\end{abstract}

Keywords: gold probe; haemostasis; injectional therapy; rebleeding; shock; peptic ulcer

Gastroenterology, Department of Medicine, Veterans General Hospital,

Taipei, Taiwan, Republic of China

$\mathrm{H}-\mathrm{J}$ Lin

G-Y Tseng

C-L Perng

F-Y Lee

F-Y Chang

S-D Lee

Correspondence to: Professor Lin.

Accepted for publication 8 December 1998 become the first line treatment of ulcer haemorrhage.

Various endoscopic treatments have been developed in the past 15 years. Heater probe thermocoagulation (HPT) and multipolar electrocoagulation are the two most promising techniques for treating peptic ulcer bleeding. ${ }^{4}$ Endoscopic injection, however, is still the most convenient and cost effective means of arresting peptic ulcer haemorrhage. ${ }^{6}$ Which of the above treatments is better at arresting peptic ulcer bleeding remains inconclusive.

Gold probe (GP) is a new catheter with bipolar electrocoagulation for peptic ulcer haemorrhage. The method of application is similar to that of conventional multipolar electrocoagulation. As a needle can be advanced at the tip of the probe, GP can be used to stop bleeding by electrocoagulation alone or by injection plus electrocoagulation (injection gold probe, IG).

The primary objective of this study was to test the null hypothesis that there was no difference in haemostatic rates among the three groups. The secondary objective was to compare the requirement for blood transfusion, surgery, mortality, and length of hospital stay among the three groups.

\section{Patients and methods}

Patients admitted with haematemesis or melaena underwent endoscopy within 24 hours of admission. Male or female patients aged 18-80 years were eligible for inclusion in this study if they presented with an actively bleeding ulcer (spurting or oozing), or a non-bleeding visible vessel (NBVV). Patients with an NBVV had to show one of the following signs of recent bleeding: coffee ground or blood in the stomach or duodenum; shock; or initial haemoglobin less than $10 \mathrm{~g} / \mathrm{l}$.

Patients presenting with any of the following were excluded: inability or unwillingness to give written informed consent; bleeding tendency (platelet count less than $50000 / \mathrm{mm}^{3}$, prothrombin time less than $30 \%$, or taking anticoagulants); bleeding gastric malignancy; pregnancy; or more than one bleeding source.

Possible complications of endoscopic treatment were discussed with the patients and their relatives and written informed consent was obtained before the trial. All haemostatic treatments were performed by HJL who had experience of at least 15000 cases in endoscopic

Abbreviations used in this paper: GP, gold probe; HPT, heater probe thermocoagulation; IG, injection gold probe; NBVV, non-bleeding visible vessel. 


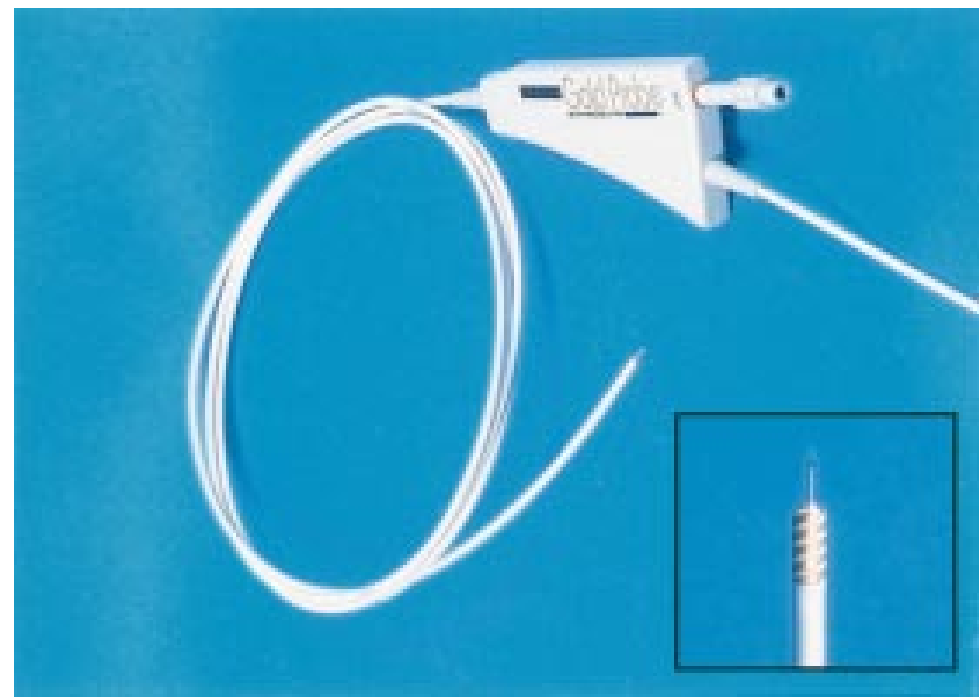

Figure 1 Gold probe: a needle can be advanced at the tip of the probe (inset).

examination and 1000 cases in endoscopic haemostasis. The study was approved by the Clinical Research Committee of the Veterans General Hospital, Taipei.

For every patient enrolled in the study, a sealed envelope was opened before performing endoscopic treatment. They were randomised to one of the following treatment groups: adrenaline group, GP group, and IG group. Randomisation was performed by a statistician who was not involved in the study.

In the adrenaline group, an Olympus PQ 20 end view endoscope and an NM-8L injector were used to perform endoscopic injection. Adrenaline 1/10 000, 0.5-1.0 ml was injected at $2-3 \mathrm{~mm}$ around the bleeder until the bleeding was controlled. In the GP group, a $7 \mathrm{Fr}$ gold probe (Microvasive, Boston Scientific Corp., USA; fig 1) was used to compress the bleeder. Thereafter, the bleeder was electrocoagulated with setting 3 for 10 seconds before moving the probe. The same procedure was repeated until haemostasis was achieved. If the bleeder could not be arrested after 30 seconds of electrocoagulation, a $10 \mathrm{Fr}$ gold probe through an Olympus 2-T end view endoscope was tried. In the IG group, adrenaline solution was injected followed by GP treatment as described earlier. The end point of treatment was defined as cessation of bleeding and flattening of the NBVV. Failure was defined for patients who did not attain initial haemostasis with the above procedure. These patients were then subjected to alternative endoscopic treatment or surgical intervention.

Rebleeding was suspected if fresh blood was found in the stomach six hours after entry into the study; if vital signs were unstable; or if there was continued tarry or bloody stools, or haematemesis. For these patients, an emergency endoscopy was performed. If active bleeding or a fresh blood clot was found at the ulcer base, rebleeding was confirmed.

For ethical reasons, treatment regimens were discussed with patients who rebled. Therapeutic options included a second treatment of the same modality, an alternative endoscopic treat- ment, or surgery. One biopsy specimen was obtained from the gastric antrum after cessation of bleeding. The presence of Helicobacter pylori was checked by a rapid urease test.

Vital signs of the patients were checked every hour for the first 12 hours, every two hours for the second 12 hours, every four hours for the following 24 hours until they became stable, and then four times daily. A nasogastric tube was inserted and maintained until 24 hours after treatment. The haemoglobin and packed cell volume were checked at least once daily, and a blood transfusion was given to maintain the haemoglobin concentration at around 10 $\mathrm{g} / \mathrm{l}$. Omeprazole $40 \mathrm{mg}$ was given intravenously every six hours for three days, then $20 \mathrm{mg}$ /day orally for two months. Triple therapy was given within 24 hours of enrolment if the urease test was positive. The physicians and surgeons were made aware of the exact endoscopic findings and treatment given in each case. Endoscopy was performed 72 hours later. If no blood clot or haemorrhage was seen in the ulcer base, the patient was discharged and followed up with an endoscopy two to three months later.

Shock was defined as systolic pressure less than $100 \mathrm{~mm} \mathrm{Hg}$ and pulse rate greater than 100 beats/min accompanied by cold sweating, pallor, and oliguria. Initial haemostasis was defined as no haemorrhage persisting for five minutes after endoscopic treatment. Ultimate haemostasis was defined as lack of rebleeding for 14 days after treatment.

The outcome assessments were initial rebleeding, number of treatment failures, requirement for blood transfusion and surgery, length of hospital stay, and mortality. Assessment of objectives was within 14 days after enrolment in the study. The sample size was calculated according to previous experience. In our previous study, the rebleeding rate following adrenaline injection was $36 \%,{ }^{7}$ and following mutipolar electrocoagulation was $7.5 \%{ }^{8}$; a sample size of 32 was thus required for each group to achieve a statistical power of $80 \%$ at $10 \%$ type I error.

One way analysis of variance (ANOVA) was used to compare age, volume of blood transfusion, volume of injected adrenaline, ulcer size, haemoglobin, and length of hospital stay. The $\chi^{2}$ test with or without Yates's correction and Fisher's exact test were used when appropriate to compare the location of bleeders, endoscopic findings, gastric contents, number of patients with Helicobacter pylori infection, shock, medical illness, haemostasis, treatment failure, emergency operation, mortality, and hospital stay among the three groups. A probability value of less than 0.05 was considered significant.

\section{Results}

Between June 1997 and April 1998, a total of 844 patients whose main symptoms were haematemesis, tarry stools, or both, attended the emergency department. A total of 740 patients received an emergency endoscopic examination within 12 hours of arrival at the emergency department. A total of 603 patients had peptic ulcers. Peptic ulcers with active bleeding or 


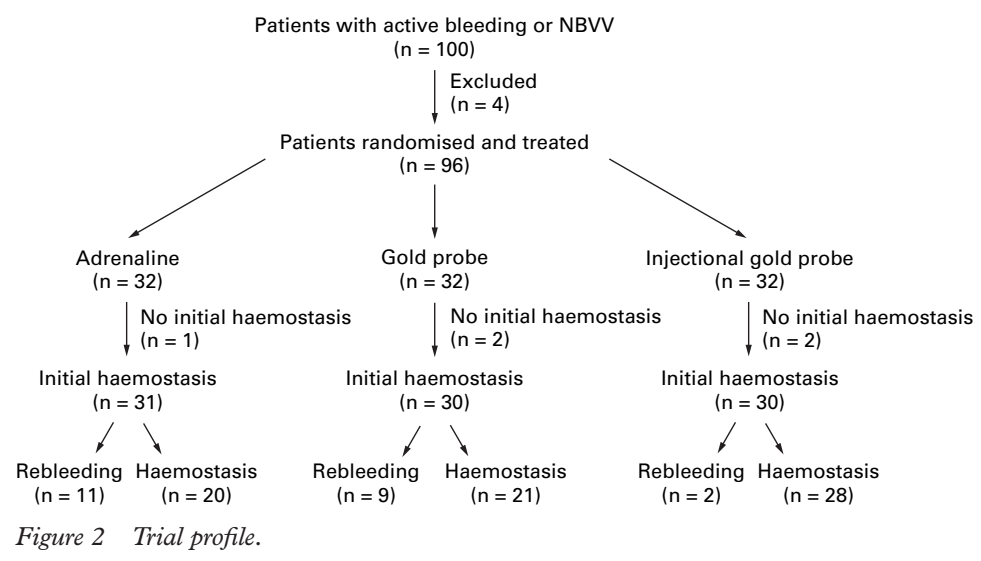

Table 1 Clinical variables of patients at entry to the study

\begin{tabular}{llll}
\hline & Adrenaline $(n=32)$ & $G P(n=32)$ & $I G(n=32)$ \\
\hline Age (mean, y) & 71.2 & 64.5 & 64.2 \\
Sex (M/F) & $29 / 3$ & $30 / 1$ & $28 / 4$ \\
Locations of bleeders & 18 & 20 & 19 \\
$\quad$ Stomach & 13 & 12 & 12 \\
$\quad$ Duodenum & 1 & 0 & 1 \\
$\quad$ Stoma & 3 & 4 & 3 \\
Endoscopic findings & 8 & 5 & 8 \\
$\quad$ Spurting & 21 & 23 & 21 \\
$\quad$ Oozing & 16 & 10 & \\
$\quad$ NBVV & 9 & 10 & 12 \\
Gastric contents & 7 & 12 & 10 \\
$\quad$ Blood & 5 & 14 & 12 \\
$\quad$ Coffee grounds & 11 & 10 & 11 \\
$\quad$ Clear & 23 & 21 & 21 \\
Positive $H$ pylori & 9.6 & 9.9 & 10 \\
No in shock & 1.0 & 1.0 & 1.1 \\
No with medical illness & & & \\
Mean haemoglobin (g/l) & Mean ulcer size (cm) & $750,490-1010$ & $556,334-778$ \\
Volume of blood transfused & & & \\
$\quad$ (ml, mean, $95 \%$ CI) & $741,467-1017$ & & \\
\hline
\end{tabular}

CI, confidence interval. patients. Five patients in the IG group and six patients in the GP group failed to achieve initial haemostasis. A 10 Fr gold probe was subsequently applied; two patients in each group failed to achieve haemostasis.

In total, initial haemostasis was not achieved in one adrenaline, two GP, and two IG patients $(p>0.1)$. There were fewer rebleeding episodes in the IG group $(2 / 30,6.7 \%)$ than in the GP group $(9 / 30,30 \%, p=0.04)$ and in the adrenaline group $(11 / 31,35.5 \%, p=0.01)$. There were fewer treatment failures (other treatment modality required) in the IG group (4/32, $12.5 \%)$ than in the adrenaline group (12/32, 37.5\%, $\mathrm{p}=0.04)$.

In the adrenaline group, the one patient who failed to achieve initial haemostasis received HPT and recovered uneventfully. Of the 11 patients who had rebleeding episodes, three underwent an emergency operation, one died after surgery, four received HPT (two recovered uneventfully, one received IG treatment and recovered uneventfully, one received emergency operation and died after surgery), and three received GP treatment (two recovered uneventfully, one underwent an operation and died after surgery).

In the GP group, the two patients who failed to achieve initial haemostasis received HPT $(n=1)$ and IG $(n=1)$ treatment and recovered uneventfully. Of the nine patients who had rebleeding episodes, one underwent an emergency operation and recovered uneventfully, two received adrenaline injection (one died of bleeding; his family refused operation due to sepsis and hypoxic encephalopathy), four received GP, and two received HPT and subsequently recovered.

In the IG group, the two patients who failed NBVV were found in 100 patients (fig 2). Four patients were excluded from the study for the following reasons: no informed consent $(n=2)$, bleeding tendency $(n=1)$, and lack of cooperation $(n=1)$. There were 96 patients enrolled in the study, 32 patients in each group. The three groups were well matched for factors affecting outcome (table 1). One bleeder was located at the posterior wall of the duodenal bulb in the adrenaline group. One bleeder in the GP group and one bleeder in the IG group were located at the lesser curvature of the high gastric body.

Table 2 shows the clinical outcome of the patients studied. The volumes of injected adrenaline were similar in both the adrenaline and IG groups. In the GP and IG groups, a 7 Fr probe was used to treat the bleeder in all to achieve initial haemostasis received HPT and recovered uneventfully. Of the two rebleeding patients, one underwent an emergency operation and died of intra-abdominal infection after surgery, the other received HPT and recovered uneventfully.

Ultimate haemostasis was obtained in the difficult to approach bleeders (one at the posterior wall of the duodenal bulb in the adrenaline group, two at the lesser curvature of the high gastric body in the GP and IG groups).

The volume of blood transfused after entry to the study was less in the IG group than in the adrenaline group $(p<0.0001)$ and in the IG group $(\mathrm{p}<0.01)$. The duration of hospital stay,

Table 2 Results of patients receiving endoscopic treatment

\begin{tabular}{llll}
\hline & Adrenaline $(n=32)$ & $G P(n=32)$ & IG $(n=32)$ \\
\hline Volume of injected adrenaline & $7.1(6.1-8.1)^{\star}$ & & $6.8(5.6-7.9)$ \\
Volume of blood transfused after entry $(\mathrm{ml})$ & $1548(846-2251)$ & $1105(574-1636)$ & $491(162-822) \dagger$ \\
No achieving initial haemostasis & 31 & 30 & 30 \\
No rebleeding & 11 & 9 & $2 \ddagger$ \\
No of treatment failures & 12 & 11 & $4 \Phi$ \\
No undergoing emergency surgery & 5 & 2 & $6.2(5.0-7.4)$ \\
Stay in hospital (days) & $8.3(6.1-10.5)$ & $8.6(6.4-10.8)$ & 1 \\
No of deaths & 3 & 1 &
\end{tabular}

* Mean (95\% confidence interval).

$t p<0.0001$ between adrenaline and IG, $p<0.01$ between GP and IG

$\neq \mathrm{p}=0.011$ between adrenaline and IG, $\mathrm{p}=0.04$ between GP and IG.

$\mathrm{S}=0.04$ between adrenaline and IG. 
number of operations, and number of deaths were not statistically different among the three groups.

No patient had perforation, aspiration pneumonia, or fever within one week after endoscopic treatment in any group.

\section{Discussion}

Death from peptic ulcer haemorrhage can be lowered with better hospital care including combined medical and surgical approaches and the latest endoscopic treatment. ${ }^{4}{ }^{9}$

As the majority of ulcer bleeding episodes stop spontaneously, endoscopic treatment should be focused on those who are prone to recurrent haemorrhage. The NIH Consensus Conference recommended that ulcers with active bleeding or NBVV should be treated endoscopically. ${ }^{4}$ Therefore, only peptic ulcer patients with active bleeding or NBVV were enrolled. In addition, the major prognostic factors (for example, age, shock, endoscopic findings, initial haemoglobin) were similar among the three groups. Interpretation of the endoscopic findings and endoscopic treatment were performed by an experienced specialist (HJL) in order to minimise the bias, although the determination of rebleeding by endoscopic criteria was subjective.

Patients with active bleeding or NBVV should be initially controlled without surgery. A skilled endoscopist is essential, and the equipment can be as simple as a standard endoscope and an injection needle. ${ }^{10}{ }^{11}$ The $\mathrm{NIH}$ Consensus Conference suggested that HPT and multipolar electrocoagulation are the two most promising methods for treating peptic ulcer bleeding. ${ }^{4}$ However, endoscopic injection is technically easier to perform and is more cost effective. In two prospective, randomised trials, endoscopic injection and multipolar electrocoagulation were concluded to be equally effective in the arrest of massive peptic ulcer bleeding. ${ }^{12}{ }^{13}$ Adrenaline injection and HPT have also been reported to be effective at stopping bleeding from actively bleeding ulcers. ${ }^{14}$ In this study, the haemostatic effect of endoscopic injection of adrenaline was comparable with that of the GP group but inferior to that of the IG group.

Although a high initial haemostatic rate can be obtained with endoscopic injection of adrenaline, the rebleeding rate after endoscopic adrenaline injection is reported to be $15-36 \% .^{7{ }^{15-17}}$ The primary mechanism of arterial haemostasis for adrenaline is compression of the artery in the serosal space. It is also associated with severe focal mucosal damage but no thrombi are found in the submucosal vessels. ${ }^{9}$ Therefore, to prevent rebleeding, it is reasonable to thrombose the vessel following injection of adrenaline. The addition of injecting sclerosant conferred no advantage in some series. ${ }^{15} 18$ In our series, the addition of sclerosant did improve rebleeding in patients with spurting haemorrhage. ${ }^{7}$ Kubba et al also confirmed that adrenaline plus thrombin injection can reduce the rebleeding rate from $20 \%$ to $4.5 \% .{ }^{19}$

The addition of contact probe or laser treatment following adrenaline injection in order to reduce the rebleeding rate is theoretically attractive. Rutgeerts et al showed that laser treatment following adrenaline injection was more effective in achieving permanent haemostasis. ${ }^{20}$ In a similar study, Loizou and Brown also confirmed a marginal improvement in primary haemostasis. ${ }^{21}$ Chung et al, in a large series, showed that the addition of heat probe treatment after adrenaline injection decreases the need for emergency surgery or blood transfusion, and reduces the hospital stay in patients with spurting haemorrhage. ${ }^{17}$ In our study, we also found that gold probe treatment following injection was better than adrenaline injection alone in reducing rebleeding.

Injection gold probe is more convenient than the conventional endoscopic injection followed by bipolar electrocoagulation because it allows thermal haemostasis and injection with a single catheter, eliminating the need for catheter exchange.

We calculated the sample size according to our previous data. ${ }^{78}$ Although the sample size of this study was smaller than those of other studies, ${ }^{14} 151922$ the result was positive. The rebleeding rate of the adrenaline group (11/31) was higher than those in the other series, ${ }^{15-17}$ but was compatible with our previous experience. ${ }^{7}$ It may be due to the fact that we had more strict criteria for patient enrolment.

In our previous study, we found that omeprazole was more effective than cimetidine in increasing intragastric $\mathrm{pH}$ and reducing rebleeding episodes in patients with bleeding ulcers after successful endoscopic treatment. ${ }^{23}$ Therefore, omeprazole was used routinely for enrolled patients in this study.

Although number of operations, mortality, and duration of hospital stay of the adrenaline and GP groups were similar to those in the IG group, it may be that aggressive endoscopic treatment was given for rebleeders in this study. Therefore, the above outcomes were similar among the three groups.

It is concluded that for patients with bleeding peptic ulcer, gold probe treatment after adrenaline injection is better than adrenaline injection or gold probe treatment alone in preventing rebleeding and decreasing the need for blood transfusion.

This work was supported by VGHTH-87-05-1, NSC87-2314B075-082, and VGH-87-10.

1 Longstreth GF. Epidemiology of hospitalization for acute upper gastrointestinal hemorrhage: a population-based upper gastrointestinal hemorrhage: a p

2 Rockall TA, Logan RFA, Devlin HB, et al. Incidence of and Rockall TA, Logan RFA, Devlin $\mathrm{HB}$, et al. Incidence of and
mortality from acute upper gastrointestinal haemorrhage in mortality from acute upper gastrointestinal haen

3 Gilbert DA. Epidemiology of upper gastrointestinal bleedGilbert DA. Epidemiology of upper gast
ing. Gastrointest Endosc 1990;36:S8-13.

4 Consensus Development Panel. Consensus statement on therapeutic endoscopy and bleeding ulcers. Gastrointest Endosc 1990;36:S62-5.

5 Cook DJ, Guyatt GH, Salena BJ, et al. Endoscopic therapy for acute nonvariceal upper gastrointestinal hemorrhage: a meta-analysis. Gastroenterology 1992;102:139-48.

6 Gralnek IM, Jensen DM, Kovacs TOG, et al. An economic analysis of patients with active arterial peptic ulcer hemorhage treated with endoscopic heater probe, injection sclehage treated with endoscopic heater probe, injection sclerosis, or surgery in a prospective,

$7 \mathrm{Lin}, \mathrm{HJ}$, Perng CL, Lee SD. Is sclerosant injection mandatory after an epinephrine injection for arrest of peptic ulcer haemorrhage? A prospective, randomized, comparative study. Gut 1993;34:1182-5. 
8 Lin HJ, Wang K, Perng CL, et al. Heater probe thermocoagulation and multipolar electrocoagulation for arrest of peptic ulcer bleeding. A prospective, randomized

9 Randall GM, Jensen DM, Hirabayashi K, et al. Controlled study of different sclerosing agents for coagulation of canine gut arteries. Gastroenterology 1989;96:1274-81.

10 Bown S. Bleeding peptic ulcers. BMF 1991;302:1417-18.

11 Lin HJ, Perng CL, Lee FY, et al. Endoscopic injection for arrest of peptic ulcer hemorrhage: final results of a prospective, randomized comparative trial. Gastrointest Endosc 1993;39:15-19.

12 Laine L. Multipolar electrocoagulation versus injection therapy in the treatment of bleeding peptic ulcers. Gastroenterology 1990:99:1303-6.

13 Waring JP, Sanowski RA, Sawyer RL, et al. A randomized comparison of multipolar electrocoagulation and injection sclerosis for the treatment of bleeding peptic ulcer. sclerosis for the treatment of
Gastrointest Endosc 1991;37:295-8.

14 Chung SCS, Leung JWC, Sung JY, et al. Injection or heat probe for bleeding ulcer. Gastroenterology 1991;100:33-7.

15 Choudari CP, Palmer KR. Endoscopic injection therapy for bleeding peptic ulcer; a comparison of adrenaline alone with adrenaline plus ethar 608-10.

16 Villanueva C, Balanzó J, Torras $\mathrm{X}$, et al. Value of second-look endoscopy after injection therapy for bleeding peptic ulcer: a prospective and randomized trial. Gastrointest Endosc 1994;40:34-9.
17 Chung SCS, Lau JYW, Sung JJY, et al. Randomised comparison between adrenaline injection alone and adrenaline injection plus heat probe treatment for actively bleeding ulcers. BMf 1997;314:1307-11.

18 Chung SCS, Leong HT, Chan ACW, et al. Epinephrine or epinephrine plus alcohol for injection of bleeding ulcers: a prospective randomised trial. Gastrointest Endosc 1996;43: 591-5.

19 Kubba AK, Murphy W, Palmer KR. Endoscopic injection for bleeding peptic ulcer: a comparison of adrenaline alone with adrenaline plus human thrombin. Gastroenterology 1996;111:623-8.

20 Rutgeerts P, Vantrappen G, Broeckaert L, et al. Comparison of endoscopic polidocanol injection and YAG laser therapy for bleeding peptic ulcers. Lancet 1989;i:1164-7.

21 Loizou LA, Bown SG. Endoscopic treatment for bleeding peptic ulcers: randomized comparison of adrenaline injection and adrenaline injection $+\mathrm{Nd}$ :YAG laser photocoagulation. Gut 1991;32:1100-3.

22 Rutgeerts P, Rauws E, Wara P, et al. Randomised trial of single and repeated fibrin glue compared with injection of polidocanol in treatment of bleeding peptic ulcer. Lancet 1997;350:692-6.

23 Lin HJ, Lo WC, Lee FY, et al. A prospective randomized comparative trial showing that omeprazole prevents rebleeding in patients with bleeding peptic ulcer after successful endoscopic therapy. Arch Int Med 1998;158:54-8. 